\title{
Medial patellofemoral ligament reconstruction: patient selection and perspectives
}

This article was published in the following Dove Press journal:

Orthopedic Research and Reviews

7 September 2017

Number of times this article has been viewed

\section{Michael R. Baer \\ Jeffrey A. Macalena}

Department of Orthopaedic Surgery, University of Minnesota, Minneapolis, MN, USA
Correspondence: Jeffrey Macalena Department of Orthopaedic Surgery, University of Minnesota, 25I2 South 7th Street, South R200, Minneapolis, MN 55454, USA

Tel +l 6122738059

Fax +l 6122737959

Email maca0049@umn.edu
Abstract: Patellofemoral instability is a painful and often recurring disorder with many negative long-term consequences. After a period of failed nonoperative management, surgical intervention has been used to reduce the incidence of patellar subluxation and dislocations. Medial patellofemoral ligament (MPFL) reconstruction successfully addresses patellofemoral instability by restoring the deficient primary medial patellar soft tissue restraint. When planning MPFL reconstruction for instability, it is imperative to consider the patient's unique anatomy including the tibial tuberosity-trochlear groove (TT-TG) distance, trochlear dysplasia, and patella alta. Additionally, it is important to individualize surgical treatment in the skeletally immature, hypermobile, and athletic populations.

Keywords: MPFL, indications, considerations, contraindications

\section{Introduction}

Patellofemoral instability is a disorder that affects the general population, with a reported primary patellar dislocation incidence rate of 5.8 per $100,000 .{ }^{1}$ However, this malady is even more common in young patients with an estimated patellar dislocation incidence rate of 29 per 100,000 in the 10-17-year age groups. ${ }^{2}$ Primary patellar dislocation has the potential to result in poor clinical outcomes including recurrent dislocation/subluxation, patellofemoral pain/anterior knee pain, impaired function, and chondral lesions as well as patellofemoral osteoarthrosis. The total redislocation rate following primary dislocation has been reported as high as $26 \%$, with a markedly increased risk of $52 \%$ in patients younger than 15 years. ${ }^{3}$ Atkin et a $l^{4}$ found that over half of their study population continued to suffer from anterior knee pain and functional impairment 6 months following a primary patellofemoral dislocation. The development of osteoarthrosis following primary dislocation is well described in the literature. ${ }^{5}$

Given this high rate of recurrence, much effort has gone into determining the risk factors for recurrent instability. In their landmark anatomic analysis, Dejour et $\mathrm{al}^{6}$ determined specific radiographic factors affecting symptomatic patellofemoral instability, with a focus on bony abnormalities including trochlear dysplasia, patella alta, and increased tibial tuberosity-trochlear groove (TT-TG) distance. This study laid the groundwork for addressing patellofemoral instability. It has been proven that the etiology of patellofemoral instability is multifactorial with both bony and soft-tissue abnormalities playing a role. ${ }^{7}$ Bony restraints include the osseous interplay of the patella within the trochlea. Soft-tissue restraints include both static and dynamic soft tissues. Soft-tissue abnormalities may be dynamic in nature, including hypermobility due to 
collagen disorders or a weakened vastus medialis obliquus (VMO) muscle, or static such as medial patellofemoral ligament (MPFL) insufficiency. ${ }^{7}$ The MPFL, a thickening of the medial retinaculum, is a major static stabilizer and has been found to be the primary medial restraint (Figure 1). The MPFL is estimated to provide $50-60 \%$ of the soft tissue restraint during the first $30^{\circ}$ of flexion prior to patellar engagement in the trochlear groove. ${ }^{8}$ An enlightening cadaver study discovered the MPFL ruptures at a mean distance of $26 \mathrm{~mm}$ of elongation, while the mean distance required for lateral patellar dislocation was $50 \mathrm{~mm} .{ }^{9}$ Consequently, MPFL rupture is present in $>90 \%$ of acute and $\sim 100 \%$ of recurrent patellar dislocations. ${ }^{10}$

Current and accepted modalities of treatment for patellofemoral instability include both nonoperative and operative treatment options determined on an individual basis following consideration of patient history, physical examination, anatomic findings, and level of activity. Nonoperative modalities include periods of immobilization, patellar stabilization with bracing or taping, activity modification and physical therapy for strengthening, and range of motion exercises. Current accepted operative interventions include lateral retinacular release, VMO advancement, MPFL imbrication, MPFL repair, as well as concomitant procedures including tibial tubercle osteotomy with distal realignment, trochleoplasty, and chondroplasty. Additionally, MPFL surgical reconstruction has been proposed in recent years as a potential solution for the problem of recurrent patellar instability. Systematic reviews have revealed substantial

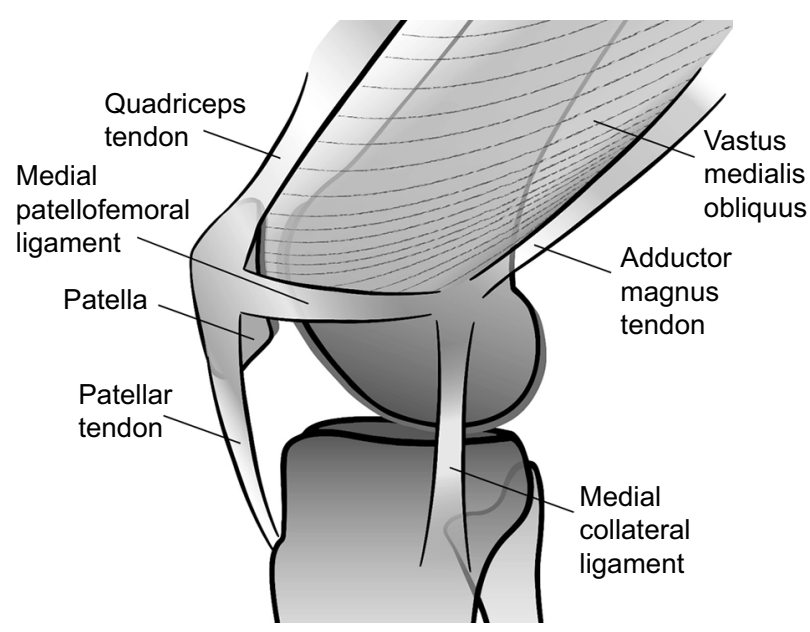

Figure I Anatomy.

Notes: Medial view of the anatomic structures of the knee (MPFL obscuring anatomy removed). Copyright (C 2017 Regents of the University of Minnesota. All rights reserved.

Abbreviation: MPFL, medial patellofemoral ligament. evidence that MPFL reconstruction is an effective procedure with encouraging outcomes. ${ }^{11-14}$ However, there remains a lack in consensus regarding appropriate patient selection for successful isolated MPFL reconstruction surgery. This review aims to provide an overview of current evidence supporting specific indications for isolated MPFL reconstruction in the setting of patellofemoral instability, important considerations for this procedure, and specific populations of which to be mindful.

\section{Indications for isolated medial patellofemoral reconstruction}

Current literature suggests that patients suffering from patellofemoral instability, ranging from recurrent patellar subluxation (apprehension) to dislocations, are the primary indication for isolated MPFL reconstruction (Table 1).

Recurrent patellofemoral instability, often without differentiation between subluxation and dislocation, has been shown to be successfully treated via isolated MPFL reconstruction. A recent systematic review discovered that $82.1 \%$ of studies included recurrent patellofemoral instability as an indication for isolated MPFL reconstruction, $33 \%$ of which failed conservative therapy. ${ }^{10}$ Moreover, the studies that specifically focused on the treatment of recurrent patellofemoral instability produced impressive clinical outcomes. Csintalan et $\mathrm{al}^{15}$ showed in their case series of 56 knees treated with MPFL reconstruction for recurrent instability that at a mean follow-up of $>4$ years there were no further patellar dislocations and subsequent subluxation occurred in only $11 \%$ of those treated. Raghuveer and Mishra $^{16}$ reported that a prospective series of 13 knees treated for generalized recurrent patellofemoral instability failed to result in a single recurrent dislocation at an average follow-up of 42 months. Similarly, Fernandez et al ${ }^{17}$ found that in their 30 knees surgically treated for recurrent instability all but one regained full range of motion within 2 months following surgery, none suffered from recurrent dislocations, and $90 \%$ had a patient-related outcome score of "excellent" at a mean follow-up of 38 months. Finally, Mikashima et al ${ }^{18}$ found that surgical treatment of 24 knees

Table I Isolated MPFL reconstruction

\begin{tabular}{l} 
Indications for isolated MPFL reconstruction \\
\hline Patellofemoral instability: \\
- Recurrent subluxation (apprehension) \\
- Recurrent dislocation
\end{tabular}

Abbreviation: MPFL, medial patellofemoral ligament. 
demonstrating recurrent instability symptoms following 3 months of conservative treatment and quadriceps muscle exercises achieved a statistically significant improvement in mean functional outcome score, with $76.5 \%$ of patients returning to preoperative sports activities at the same level at an average follow-up of 41 months.

Additionally, a single patellar dislocation with ongoing subluxation symptoms (lateral excursion of the patella without dislocation; clinically described as apprehension) has been shown to be successfully treated with isolated MPFL reconstruction. Howells et al ${ }^{12}$ reported the clinical outcomes of a prospective analysis of 211 knees in patients treated with MPFL reconstruction, six (2.8\%) of which had a surgical indication of a single dislocation with ongoing symptomatic instability. Although inferior in number when compared to the 141 knees in the cohort treated for atraumatic recurrent patellar dislocation, analysis failed to reveal a statistically significant difference in postoperative outcome between the particular indications. The prospective outcome assessment found no recurrent subluxation or dislocations at a mean follow-up of 16 months. Furthermore, there was a statistically significant improvement in available pre- and postoperative outcome scores in both groups. ${ }^{12} \mathrm{Ma}$ et al published a prospective randomized comparative trial for patients either with one patellar dislocation with recurrent instability symptoms or with multiple dislocations. The 32 patients who underwent MPFL reconstruction had significantly improved average functional outcome scores as well as a decreased patellar lateral shift at a mean followup of 40 months. ${ }^{19}$

Multiple patellar dislocations, most commonly noted as a minimum of two or more, have also been shown to be a successful indication for isolated MPFL reconstruction. Lippacher et al reviewed a case series of young patients suffering from recurrent patellar dislocations. They found that $100 \%$ of patients who participated in sports preoperatively returned to sports following surgery in a cohort of 72 knees of patients with two or more patellar dislocations despite a nonoperative treatment program. Moreover, $\sim 80 \%$ of the patients rated themselves as "very satisfied" or "satisfied" with the results and there were significant improvements in patient-reported outcomes and pain scores. ${ }^{20}$ Christiansen et $\mathrm{al}^{21}$ presented a case series of 44 patients who underwent surgical correction for recurrent patellar dislocations and found that, at a mean follow-up of 22 months, subjective patient-reported outcomes revealed that $80 \%$ of surgical patients felt that their activity of daily living knee function had improved, with only one redislocation, and persistent chronic pain remained in only the four subjects with high grade preoperative cartilage injury to the patella or trochlea. Additionally, multiple studies found similar success with an indication of three or more patellar dislocations. Ronga et $\mathrm{al}^{22}$ described a prospective case series in which 28 people underwent surgery for three or more patellar dislocations following intensive conservative rehabilitation programs, revealing statistically significant increases in the mean functional outcome scores, with only three patients experiencing a recurrent patellar dislocation at an average clinical follow-up of 3 years.

As presented, isolated MPFL reconstruction has the potential to successfully treat recurrent patellofemoral instability, resulting in the prevention of further patellar subluxation, dislocations, and improved overall knee function.

\section{Considerations}

As previously described, patellofemoral instability is multifactorial in nature. Historically, there are certain scenarios in which isolated MPFL reconstruction is not recommended. Common contraindication scenarios noted in literature include situations of bony malalignment, trochlear dysplasia, and patella alta (Table 2).

An enlarged lateral distance between the position of the tibial tubercle relative to the central trochlear groove is a commonly cited contraindication to isolated MPFL reconstruction surgery for patellofemoral instability. This relationship, as represented by the TT-TG distance, is important for the assessment of the laterally directed force on the extensor mechanism. The TT-TG distance is a measure (reported in millimeter) of the lateralization of the tibia in reference to the femoral groove and can be measured via superimposed axial computed tomography (CT) or magnetic resonance (MR) images that include the tibial tuberosity and deepest point of the trochlear groove (Figure 2) ${ }^{6,23}$ TT-TG distances $<12 \mathrm{~mm}$ are normal, whereas values $>20$ are associated with increased risk of recurrent instability. ${ }^{6,24}$ Although there is limited data supporting q-angle in relation to isolated MPFL repair and clinical outcomes, a prospective study of people with patellofemoral symptoms concluded that there is a statistically significant and reliable correlation between

Table 2 Common contraindication scenarios

\begin{tabular}{ll}
\hline Considerations & \\
\hline I. Enlarged TT-TG distance & Tibial tubercle medialization \\
2. Trochlear dysplasia & Deepening trochleoplasty \\
3. Patella alta & Tibial tubercle distalization \\
\hline Abbreviation: TT-TG, tibial tuberosity-trochlear groove.
\end{tabular}




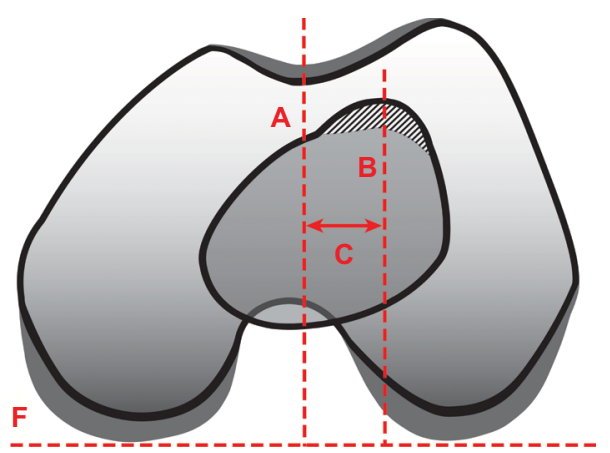

Figure 2 Tibial tuberosity-trochlear groove measurement.

Notes: Tibial tuberosity-trochlear groove distance (measured via superimposed axial images) $=\mathrm{C}$. Baseline $\mathrm{F}$ measured along posterior femoral condyles. Line $\mathrm{A}$ is perpendicular to baseline $\mathrm{F}$ and bisects the deepest point of the trochlear groove. Line $B$ is perpendicular to baseline $F$, and therefore parallel to line $A$, and bisects the midline of the tibial tuberosity. Distance $C$ is the measured distance between lines $A$ and B. Copyright (C) 2017 Regents of the University of Minnesota. All rights reserved.

the q-angle and the TT-TG distance. ${ }^{25}$ As a result of the determined historic pathologic threshold, an increased TTTG distance has been suggested as an indication for tibial tuberosity medialization in order to address knee pain and the increased risk of patellofemoral instability. ${ }^{26}$ As a result, many surgeons recommend a medial tibial tuberosity transfer in addition to MPFL reconstruction for patellofemoral instability if the patient's TT-TG distance is $>15 \mathrm{~mm} \cdot{ }^{27,28}$ This is reiterated in literature, as isolated MPFL reconstruction studies commonly include a TT-TG distance exclusion criteria benchmark of $>15 \mathrm{~mm}$, with others citing up to $22 \mathrm{~mm}$ as a cutoff point. ${ }^{10}$ However, a recent case-control study of 34 knees that underwent MPFL reconstruction for recurrent patellar dislocation failed to find a significant correlation between TT-TG distance and postoperative clinical scores following surgery between the control TT-TG distance group (mean distance $15.7 \mathrm{~mm}$ ) and the increased TT-TG distance group (mean distance $22.7 \mathrm{~mm}$ ) at an average follow-up of 3 years. ${ }^{23}$ Therefore, although it is important to consider a TT-TG distance of $>15-20 \mathrm{~mm}$ when planning an isolated MPFL reconstruction for patellofemoral instability, further research is needed in this area.

Another common contraindication for an isolated MPFL reconstruction is the presence and the grade of trochlear dysplasia. The trochlea is normally concave in nature. However, in cases of dysplasia, the trochlea has abnormal morphology defined by a shallow and shortened trochlear groove with resultant decreased bony constraint of the patella. As a result, trochlear dysplasia as an anatomic variant is considered as a predisposing risk factor for recurrent patellar dislocation and has therefore been cited as an indication for combined MPFL reconstruction and deepening trochleoplasty procedures. ${ }^{29}$
Dejour et al originally referred to trochlear dysplasia as the "fundamental factor" as it was identified in $85 \%$ of study patients with patellar instability. The group developed a classification system representing low-, moderate-, and highgrade dysplasia. ${ }^{6}$ This classification, defined by evaluation of true lateral radiographs, was later modified into grades A-D with the addition of axial imaging (Figure 3 ). ${ }^{30}$ For improved interobserver reliability, this classification system has been further simplified to low-grade trochlear dysplasia (Dejour grade A) and high-grade trochlear dysplasia (Dejour grades $\mathrm{B}, \mathrm{C}$, and D). ${ }^{31}$ Traditionally, trochlear dysplasia has been noted as a contraindication for isolated MPFL reconstruction as measured by both the Dejour classification and the trochlear-sulcus angle (angle between the slopes of the medial and lateral trochlea as seen via radiographic Merchant view). Dejour classification contraindications cited in literature include greater than or equal to grade $\mathrm{B}$, greater than grade B, or simply "high-grade" trochlear dysplasia. ${ }^{10}$ Trochlear-sulcus contraindications include both angles $>145$ and $150^{\circ} .{ }^{10}$ Hiemstra et al recently reported on a cohort of 277 patellofemoral stabilization procedures, 152 of which were MPFL reconstructions, in an attempt to determine whether an isolated soft tissue procedure for patellofemoral instability would be successful in patients with high-grade dysplasia. Although there was an overall increase in postoperative disease-specific quality of life and pain scores in all patients who underwent stabilization procedures, their examination revealed a statistically significant negative correlation between the presence of high-grade (Dejour grades

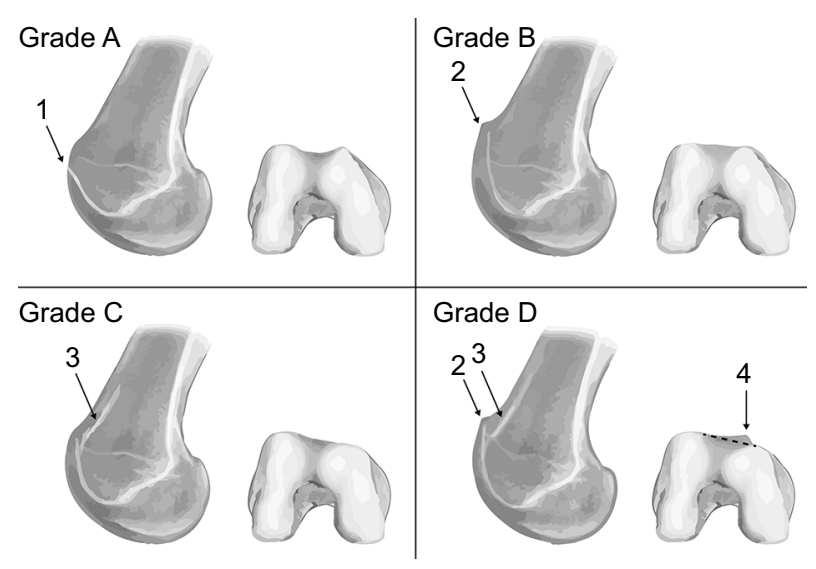

Figure 3 Dejour trochlear dysplasia classification.

Notes: Dejour trochlear dysplasia grade $A$ is defined by a crossing sign (I) on lateral radiograph and a shallow trochlear bony sulcus on axial imaging (angle $>145^{\circ}$ ). Grade $B$ is defined by a supratrochlear spur (2) on lateral radiograph and a flat trochlea on axial imaging. Grade $C$ is defined by a double contour (3) on lateral radiograph, medial condyle hypoplasia, and lateral trochlear convexity on axial imaging. Grade D is defined by both a double contour (3) and supratrochlear spur (2) seen on lateral radiograph and severe trochlear asymmetry with a "cliff' (4) separating the medial and lateral condyles. Copyright $@$ Regents of the University of Minnesota. All rights reserved. 
B-D) trochlear dysplasia and clinical outcome scores at an average of 2 years following isolated MPFL reconstruction. ${ }^{29}$ In addition, a recent systematic review found that despite current literature reporting that both trochleoplasty and MPFL reconstruction have similar abilities to produce good clinical outcomes for patellofemoral instability, an isolated MPFL procedure without correction of severe trochlear dysplasia (Dejour grades B-D) via trochleoplasty resulted in a significantly increased likelihood of postoperative patella redislocation or subluxation. ${ }^{27}$ Therefore, although it appears appropriate to be wary of severe trochlear dysplasia when planning an isolated MPFL reconstruction, future research is needed in this area.

Patella alta is described as a predisposing risk factor for recurrent patellofemoral instability and dislocations. ${ }^{32-34}$ Therefore, isolated MPFL reconstruction for recurrent patellofemoral instability is commonly contraindicated in the setting of profound patella alta. The mechanical consequence of this condition includes a delay in patella interaction with the trochlear groove during flexion, thereby increasing the amount of flexion required to achieve patella stability within the patellofemoral joint. This delay provides a larger range of knee motion without trochlear resistance to lateral patellar translation. Patella alta is reported to be present in $24 \%$ of patients with intermittent patellar dislocation and has been mentioned as a predictor for recurrent instability in patients treated with isolated MPFL reconstruction. ${ }^{6,35}$ However, results for isolated MPFL reconstruction for patella alta are rarely reported. ${ }^{36} \mathrm{~A}$ number of methods for assessing patellar height have been described, including those relating the position of the patella to the femur (direct) and those relating it to the tibia (indirect). The two most widely accepted radiographic techniques were described by Insall-Salvati (IS) and Caton-Deschamps (CD) as the ratio between the length of the patellar tendon and patella and as the ratio of the distance from the inferior margin of the articular surface of the patella and the anterosuperior angle of the tibial plateau and the length of the patellar joint surface, respectively (Figure 4). ${ }^{37}$ The normal IS ratio range is from 0.8 to 1.2 , with patella alta $>1.2$. The normal $\mathrm{CD}$ ratio range is from 0.6 to 1.3 , with patella alta $>1.3 .{ }^{37}$ Patella alta is a frequently cited contraindication or exclusion criterion for isolated MPFL reconstruction for patellofemoral instability, with the majority reporting a cutoff threshold of either an IS ratio or a $C D$ ratio of $>1.2 .{ }^{10}$ However, there are references to patella alta cutoff values of IS ratios $>1.3$ or CD ratios $>1.35$ as well. ${ }^{10}$ The primarily described technique for addressing patella alta in a skeletally mature patient in the setting of

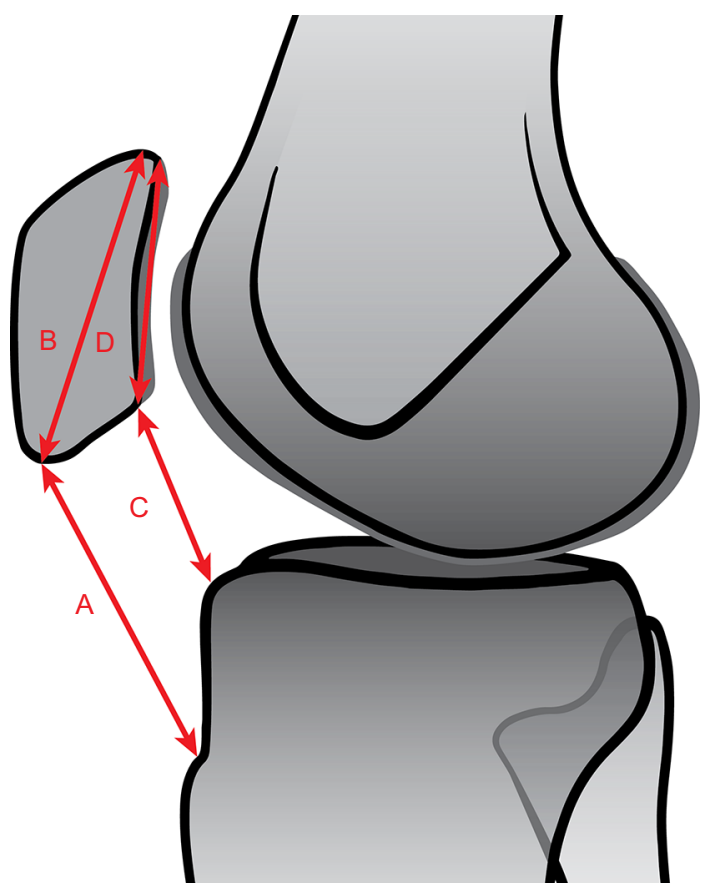

Figure 4 Insall-Salvati and Caton-Deschamps measurements for patella alta. Notes: Insall-Salvati measurement for patella alta $=A / B$. Line $A$ is measured from the point of patella insertion on the inferior patella to the superior aspect of patellar tendon insertion on the tibial tuberosity. Line $B$ is measured from the most superior subchondral bone to the point of patellar tendon insertion on the inferior patella. Caton-Deschamps measurement for patella alta $=C / D$. Line $C$ is measured from the inferior aspect of patellar cartilage articular surface to the anterior corner of the superior tibial joint surface. Line $D$ is measured from superior to inferior aspects of patellar cartilage articular surface. Copyright @ 2017 Regents of the University of Minnesota. All rights reserved.

patella instability is a tibial tubercle distalization. Lowering the patella height produces greater contact between the patella and trochlea, thereby improving bony resistance to lateral translation of the patella. ${ }^{38}$ A systematic review by Magnussen et al revealed tibial tubercle distalization as an effective approach to normalizing patellar height in patients with instability. In the five studies reviewed, there was a very low risk of reported recurrent patellar dislocation (overall risk of $1.75 \%$ ). However, patellar apprehension was found in $15-33 \%$ of patients at final follow-up (overall risk of $26.3 \%$ ). The authors concluded that although distalization of the tibial tubercle should be considered for patients with significant patella alta (CD index $>1.2$ ) and patellofemoral instability, supplementation with a soft tissue procedure (such as MPFL reconstruction) should be considered to reduce the risk of persistent subtle postoperative patellar instability. ${ }^{38}$ Therefore, although patella height has historically been shown to play a role in patellofemoral instability and is an important consideration when planning an isolated MPFL reconstruction for patellofemoral instability, further research is needed in this area. 
As important as it is to recognize these anatomical scenarios, it is just as important to understand that they are not mutually exclusive. In a prospective study, Wagner et al evaluated 50 patients for coexisting risk factors for patellofemoral instability. The study found that $98 \%$ of included patients had some level of trochlear dysplasia, while 58\% had patella alta (IS >1.2) and 36\% had TT-TG distances $>15 \mathrm{~mm} .{ }^{39}$ As such, it may be essential to address multiple causes of instability with additional procedures as trochlear dysplasia, an enlarged TT-TG distance and patella alta frequently occur simultaneously.

\section{Special populations}

As described, there are multiple anatomic and biomechanic considerations to keep in mind when planning an isolated MPFL reconstruction for patellofemoral instability. Furthermore, it is important to contemplate the patient's skeletal maturity, potential presence of joint hypermobility, as well as their desire for return to athletic participation following surgery in order to provide the best individual outcomes (Table 3).

Skeletally immature patients frequently encounter recurrent patellar instability. As in adults, patellofemoral instability is commonly multifactorial in etiology with both osseous and soft tissue contributions. Therefore, it is again important to evaluate for bony malalignment, trochlear dysplasia, and patella alta. Similarly, surgical reconstruction of the MPFL has produced promising results for the pediatric patient with recurrent patellar instability following failure of conservative management. ${ }^{40}$ However, when implementing this technique, it is important to consider the close anatomic proximity of the medial distal femoral insertion of the MPFL and the distal femoral physis, as it is responsible for $\sim 70 \%$ of femoral growth and $\sim 37 \%$ of total lower extremity growth. ${ }^{41-43}$ It is imperative to consider this anatomy in the setting of skeletal immaturity in order to prevent iatrogenic growth plate injury or arrest while drilling the femoral tunnel during MPFL reconstruction. Multiple studies have shown that femoral drilling is a safe and suitable treatment option in the skeletally immature. ${ }^{44,45}$ If concern for physeal injury remains, successful outcomes

Table 3 Special populations

Special populations

I. Skeletal immaturity

2. Hypermobility

3. Athlete have been reported without the use of drilling via a slightly less anatomic MPFL reconstruction. ${ }^{46}$ Noyes and Albright reported on the utilization of the medial aspect of the quadriceps tendon with its retinacular patellar attachment intact to avoid patellar and femoral drill holes. ${ }^{47}$ Additionally, nonanatomic procedures have been described with impressive success rates. ${ }^{48,49}$ Marsh et $\mathrm{al}^{50}$ reported excellent results in $>86 \%$ of adolescent patients with chronic recurrent patellar instability treated with a modified Roux-Goldthwait procedure, involving the longitudinal split, transfer, and reattachment of the lateral patella tendon distally and medially to the soft tissues on the medial side of the tibia. Isolated MPFL reconstruction is a suitable treatment option for recurrent patellofemoral instability in the pediatric population; however, it is important to be aware of the distal femoral physis during anatomic or nonanatomic reconstruction procedures.

With the increasingly popular use of MPFL reconstruction for patellar instability, it is important to understand what role generalized hypermobility plays in clinical outcomes. People with joint hypermobility are generally asymptomatic; however, some may present with pain and discomfort attributable to their condition. Symptomatic hypermobility is estimated to occur in $10-20 \%$ of the general population, with higher percentages seen in Indian, Chinese, and Middle Eastern populations. ${ }^{51}$ Howells and Eldridge investigated the influence of hypermobility on the outcome of an isolated MPFL reconstruction for patients with patellar instability. The group described a series of 25 patients who all met preoperative Beighton criteria for hypermobility as well as a matched cohort of 50 patients by age, gender, indication for surgery, and degree of trochlear dysplasia without hypermobility. ${ }^{52,53}$ At a mean follow-up of 16 months, the hypermobile group had a significant overall postoperative improvement in function. However, the hypermobile patients had significantly worse postoperative functional outcomes when compared with the control group, including an increased rate of residual and recurrent symptoms and a lower rate of return to sport. ${ }^{52}$ Isolated MPFL reconstruction is a reasonable and effective treatment option for patients with generalized hypermobility; however, individualized counseling on expectations is important.

Finally, it is important to consider the desire for return to sport or athletic participation following isolated MPFL reconstruction for recurrent patellar instability. Until recently, information has been limited regarding MPFL reconstruction and return to play. ${ }^{54}$ The previously mentioned prospective series by Howells and Eldridge ${ }^{52}$ 
reported that $76 \%$ of their patients treated with isolated MPFL reconstruction for patellofemoral instability resumed sporting activities at 16 months follow-up, although the specific activity level was not described. Additionally, Panni et al ${ }^{55}$ described a prospective case series of 45 athletically active patients treated with MPFL reconstruction for recurrent patellar dislocation, revealing $64 \%$ of patients returning to similar sports or level of activity, $16 \%$ of patients returning to a reduced level of activity or a change in the type of sport secondary to reasons unrelated to surgery, and $20 \%$ of patients returning to reduced levels or a change in sport as a result of surgery at an average follow-up of 33 months. In addition, Lippacher et al reported on isolated MPFL reconstructions in a cohort including both adolescents and adults (age range 14.8-43.8 years, median age 18.3 years) with exclusion of patients with severe trochlear dysplasia and increased TT-TG distances to avoid potential confounding bias. At a minimum postoperative follow-up of 2 years, the study revealed both statistically significant improvements in all knee outcome scores as well as a return to sporting activities for all patients involved in sports preoperatively. ${ }^{20}$ The group also found that $53 \%$ of patients involved in sports preoperatively returned to the same level following surgery, with $47 \%$ returning to lower activity levels. ${ }^{20}$ Of those that returned to lower activity levels, $48 \%$ cited a personal desire to avoid excessive sports following surgery for fear of reinjury, $27 \%$ with reasons unrelated to surgery, and $24 \%$ as a result of decreased knee function. ${ }^{20}$ The study reported comparable return-to-sports rates after isolated MPFL reconstruction with ACL reconstruction, high tibial osteotomy, and articular cartilage repair outcome studies. ${ }^{20}$ Therefore, isolated MPFL reconstruction is an effective surgery for patellofemoral instability with regard to return to sports activities postoperatively.

\section{Conclusion}

MPFL reconstruction has become a popular means to address patellofemoral instability, with systematic review revealing that an isolated procedure is an effective treatment option providing both subjective and functional improvements. ${ }^{52,54,56,57}$ Although this procedure has been shown to successfully treat those with recurrent patellofemoral subluxation and dislocations, there remains a lack of agreement regarding specific situations requiring additional procedures to address confounding static and dynamic contributors to the instability of the patellofemoral joint. A recent systematic review by Longo et al attempted to analyze the current indications, outcomes, and complication rates of MPFL reconstruction associated with additional bony procedures. The author's review of the relevant literature revealed consistent consideration of tibial trochlear groove distance, trochlear dysplasia, and patellar height when planning surgical management for patellofemoral instability. However, there was no consensus on specific threshold values in order to guide surgical planning with regard to MPFL reconstruction with or without a combined subsequent procedure. ${ }^{57} \mathrm{~A}$ recent attempt to establish an algorithmic approach to managing recurrent lateral patellar dislocation by Weber et al $^{58}$ recommends anatomic MPFL reconstruction in the setting of a disrupted ligament, combined with tibial tubercle realignment in the setting of increased TTTG distance and/or patella alta, as well as a groove-deepening trochleoplasty for trochlear dysplasia grade B or grade D. However, the authors admit that the lack of definitive evidence supporting consistent surgical algorithms as the available data remains largely level IV. ${ }^{58}$ Patellofemoral instability and surgical indications would greatly benefit from prospective, randomized controlled trials with larger patient populations in order to collect impactful outcome measures. Therefore, although an isolated MPFL reconstruction is generally an acceptable and effective treatment option for patellofemoral instability, surgical management should be individualized in order to assess and address the multifactorial nature of the malady, including the TT-TG distance, trochlear dysplasia, and patella alta. In addition, it is important to assess skeletal maturity, preoperatively identify hypermobility, and discuss return to play with the athletic patient.

\section{Disclosure}

The authors report no conflicts of interest in this work.

\section{References}

1. Fithian DC, Paxton EW, Stone ML, et al. Epidemiology and natural history of acute patellar dislocation. Am J Sports Med. 2004;32(5):1114-1121.

2. Mehta VM, Inoue M, Nomura E, Fithian DC. An algorithm guiding the evaluation and treatment of acute primary patellar dislocations. Sports Med Arthrosc. 2007;15(2):78-81.

3. Buchner M, Baudendistel B, Sabo D, Schmitt H. Acute traumatic primary patellar dislocation: long-term results comparing conservative and surgical treatment. Clin J Sport Med. 2005;15:62-66.

4. Atkin DM, Fithian DC, Marangi KS, Stone ML, Dobson BE, Mendelsohn C. Characteristics of patients with primary acute lateral patellar dislocation and their recovery within the first 6 months of injury. Am J Sports Med. 2000;28(4):472-479.

5. Mäenpää H, Lehto MU. Patellofemoral osteoarthritis after patellar dislocation. Clin Orthop Relat Res. 1997;(339):156-162.

6. Dejour H, Walch G, Nove-Josserand L, Guier C. Factors of patellar instability: an anatomic radiographic study. Knee Surg Sports Traumatol Arthrosc. 1994;2(1):19-26.

7. Colvin AC, West RV. Patellar instability. J Bone Joint Surg Am. 2008;90(12):2751-2762. 
8. Philippot R, Boyer B, Testa R, Farizon F, Moyen B. The role of the medial ligamentous structures on patellar tracking during knee flexion. Knee Surg Sports Traumatol Arthrosc. 2012;20(2):331-336.

9. Mountney J, Senavongse W, Amis AA, Thomas NP. Tensile strength of the medial patellofemoral ligament before and after repair or reconstruction. J Bone Joint Surg. 2005;87(1):36-40.

10. Yeung M, Leblanc M-C, Ayeni OR, et al. Indications for medial patellofemoral ligament reconstruction: a systematic review. J Knee Surg. 2016;29(7):543-554.

11. Buckens CFM, Saris DBF. Reconstruction of the medial patellofemoral ligament for treatment of patellofemoral instability: a systematic review. Am J Sports Med. 2010;38(1):181-188.

12. Howells NR, Barnett AJ, Ahearn N, Ansari A, Eldridge JD. Medial patellofemoral ligament reconstruction: a prospective outcome assessment of a large single centre series. J Bone Joint Surg. 2012;94(9):1202-1208.

13. Russ SD, Tompkins M, Nuckley D, Macalena J. Biomechanical comparison of patellar fixation techniques in medial patellofemoral ligament reconstruction. Am J Sports Med. 2015;43(1):195-199.

14. Smith TO, Walker J, Russell N. Outcomes of medial patellofemoral ligament reconstruction for patellar instability: a systematic review. Knee Surg Sports Traumatol Arthrosc. 2007;15(11):1301-1314.

15. Csintalan RP, Latt LD, Fornalski S, Raiszadeh K, Inacio MC, Fithian DC. Medial patellofemoral ligament (MPFL) reconstruction for the treatment of patellofemoral instability. J Knee Surg. 2014;27(2):139-146.

16. Raghuveer RK, Mishra CB. Reconstruction of medial patellofemoral ligament for chronic patellar instability. Indian J Orthop. 2012;46(4):447-454.

17. Fernandez E, Sala D, Castejon M. Reconstruction of the medial patellofemoral ligament for patellar instability using a semitendinosus autograft. Acta Orthop Belg. 2005;71(3):303-308.

18. Mikashima Y, Kimura M, KobayashiY, Miyawaki M, Tomatsu T. Clinical results of isolated reconstruction of the medial patellofemoral ligament for recurrent dislocation and subluxation of the patella. Acta Orthop Belg. 2006;72(1):65-71.

19. Ma LF, Wang F, Chen BC, Wang CH, Zhou JW, Wang HY. Medial retinaculum plasty versus medial patellofemoral ligament reconstruction for recurrent patellar instability in adults: a randomized controlled trial. Arthroscopy. 2013;29(5):891-897.

20. Lippacher S, Dreyhaupt J, Williams SRM, Reichel H, Nelitz M. Reconstruction of the medial patellofemoral ligament: clinical outcomes and return to sports. Am J Sports Med. 2014;42(7):1661-1668.

21. Christiansen SE, Jacobsen BW, Lund B, Lind M. Reconstruction of the medial patellofemoral ligament with gracilis tendon autograft in transverse patellar drill holes. Arthroscopy. 2008;24(1):82-87.

22. Ronga M, Oliva F, Longo UG, Testa V, Capasso G, Maffulli N. Isolated medial patellofemoral ligament reconstruction for recurrent patellar dislocation. Am J Sports Med. 2009;37(9):1735-1742.

23. Matsushita T, Kuroda R, Oka S, Matsumoto T, Takayama K, Kurosaka M. Clinical outcomes of medial patellofemoral ligament reconstruction in patients with an increased tibial tuberosity-trochlear groove distance. Knee Surg Sports Traumatol Arthrosc. 2014;22(10):2438-2444.

24. Conlan T, Garth WP, Lemons JE. Evaluation of the medial soft-tissue restraints of the extensor mechanism of the knee. J Bone Joint Surg. 1993;75(5):682-693.

25. Dickschas J, Harrer J, Bayer T, Schwitulla J, Strecker W. Correlation of the tibial tuberosity-trochlear groove distance with the Q-angle. Knee Surg Sports Traumatol Arthrosc. 2016;24(3):915-920.

26. Tecklenburg K, Feller JA, Whitehead TS, Webster KE, Elzarka A. Outcome of surgery for recurrent patellar dislocation based on the distance of the tibial tuberosity to the trochlear groove. J Bone Joint Surg. 2010;92(10):1376-1380.

27. Balcarek P, Jung K, Ammon J, et al. Anatomy of lateral patellar instability: trochlear dysplasia and tibial tubercle-trochlear groove distance is more pronounced in women who dislocate the patella. Am J Sports Med. 2010;38(11):2320-2327.

28. Koëter S, Diks MJ, Anderson PG, Wymenga AB. A modified tibial tubercle osteotomy for patellar maltracking: results at two years. J Bone Joint Surg. 2007;89:180-185.
29. Hiemstra LA, Kerslake S, Loewen M, Lafave M. Effect of trochlear dysplasia on outcomes after isolated soft tissue stabilization for patellar instability. Am J Sports Med. 2016;44(6):1515-1523.

30. Tecklenburg K, Dejour D, Hoser C, Fink C. Bony and cartilaginous anatomy of the patellofemoral joint. Knee Surg Sports Traumatol Arthrosc. 2006;14(3):235-240.

31. Lippacher S, Dejour D, Elsharkawi M, et al. Observer agreement on the Dejour Trochlear dysplasia classification: a comparison of true lateral radiographs and axial magnetic resonance images. Am J Sports Med. 2012;40(4):837-843.

32. Beasley LS, Vidal AF. Traumatic patellar dislocation in children and adolescents: treatment update and literature review. Curr Opin Pediatr. 2004;16(1):29-36.

33. Hawkins RJ, Bell RH, Anisette G. Acute patellar dislocations: the natural history. Am J Sports Med. 1986;14(2):117-120.

34. McManus F, Rang M, Heslin DJ. Acute dislocation of the patella in children: the natural history. Clin Orthop Relat Res. 1979;139:88-91.

35. Singerman R, Davy DT, Goldberg VM. Effects of patella alta and patella infera on patellofemoral contact forces. J Biomech. 1994;27(8):1059-1065.

36. Tompkins MA, Arendt EA. Patellar instability factors in isolated medial patellofemoral ligament reconstructions - what does the literature tell us? A systematic review. Am J Sports Med. 2015;43(9):2318-2327.

37. Phillips CL, Silver DA, Schranz PJ, Mandalia V. The measurement of patellar height: a review of the methods of imaging. J Bone Joint Surg Br. 2010;92(8):1045-1053.

38. Magnussen RA, De Simone V, Lustig S, Neyret P, Flanigan DC. Treatment of patella alta in patients with episodic patellar dislocation: a systematic review. Knee Surg Sports Traumatol Arthrosc. 2014;22(10): 2545-2550

39. Wagner D, Pfalzer F, Hingelbaum S, Huth J, Mauch F, Bauer G. The influence of risk factors on clinical outcomes following anatomical medial patellofemoral ligament (MPFL) reconstruction using the gracilis tendon. Knee Surg Sports Traumatol Arthrosc. 2013;21(2): 318-324.

40. May MM, Parikh SN, Wall EJ, et al. Medial patellofemoral ligament surgery in the skeletally immature patient. Is reconstruction better than repair? POSNA Annual Meeting. Waikoloa Hawaii: 2010.

41. Dimeglio A. Growth in pediatric orthopaedics. In: Morrissy RT, Weinstein SL, editors. Lovell and Winter's Pediatric Orthopaedics. Philadelphia: Lippincott Williams \& Wilkins; 2005:35-65.

42. Shea KG, Grimm NL, Belzer J, Burks RT, Pfeiffer R. The relation of the femoral physis and the medial patellofemoral ligament. Arthroscopy. 2010;26:1083-1087.

43. Nelitz M, Dornacher D, Dreyhaupt J, et al. The relation of the distal femoral physis and the medial patellofemoral ligament. Knee Surg Sports Traumatol Arthrosc. 2011;19:2067-2071.

44. Kepler CK, Bogner EA, Hammoud S, Malcolmson G, Potter HG, Green DW. Zone of injury of the medial patellofemoral ligament after acute patellar dislocation in children and adolescents. Am J Sports Med. 2011;39(7):1444-1449.

45. Vavken P, Wimmer MD, Camathias C, Quidde J, Valderrabano V, Pagenstert G. Treating patella instability in skeletally immature patients. Arthroscopy. 2013;29(8):1410-1422.

46. Sillanpää PJ, Mäenpää HM, Mattila VM, Visuri T, Pihlajamäki H. A mini-invasive adductor magnus tendon transfer technique for medial patellofemoral ligament reconstruction: a technical note. Knee Surg Sports Traumatol Arthrosc. 2009;17(5):508-512.

47. Noyes FR, Albright JC. Reconstruction of the medial patellofemoral ligament with autologous quadriceps tendon. Arthroscopy. 2006;22(8):. e1-.e7.

48. Baker RH, Carroll N, Dewar FP, Hall JE. The semitendinosus tenodesis for recurrent dislocation of the patella. J Bone Joint Surg. 1972;54(1):103-109.

49. Fondren FB, Goldner JL, Bassett FH $3^{\text {rd }}$. Recurrent dislocation of the patella treated by the modified Roux-Goldthwait procedure. A prospective study of forty-seven knees. J Bone Joint Surg Am. 1985;67(7): 993-1005. 
50. Marsh JS, Daigneault JP, Sethi P, Polzhofer GK. Treatment of recurrent patellar instability with a modification of the Roux-Goldthwait technique. J Pediatr Orthop. 2006;26(4):461-465.

51. Ross J, Grahame R. Joint hypermobility syndrome. BMJ. 2011;342: 275-277.

52. Howells NR, Eldridge JD. Medial patellofemoral ligament reconstruction for patellar instability in patients with hypermobility a case control study. J Bone Joint Surg. 2012;9494(12):1655-1659.

53. Smits-Engelsman B, Klerks M, Kirby A. Beighton score: A valid measure for generalized hypermobility in children. J Pediatr. 2011;158(1):119-123, 123.e1-4

54. Fisher B, Nyland J, Brand E, Curtin B. Medial patellofemoral ligament reconstruction for recurrent patella dislocation: a systematic review including rehabilitation and return-to-sports efficacy. Arthroscopy. 2010;26(10):1384-1394.
55. Panni AS, Alam M, Cerciello S, Vasso M, Maffulli N. Medial patellofemoral ligament reconstruction with a divergent patellar transverse 2-tunnel technique. Am J Sports Med. 2011;39(12):2647-2655.

56. Schepsis AA, Rogers AJ. Medial patellofemoral ligament reconstruction: indications and technique. Sports Med Arthrosc. 2012;20(3): $162-170$.

57. Longo UG, Berton A, Salvatore G, et al. Medial patellofemoral ligament reconstruction combined with bony procedures for patellar instability: current indications, outcomes, and complications. Arthroscopy. 2016;32(7):1421-1427.

58. Weber AE, Nathani A, Dines JS, et al. An algorithmic approach to the management of recurrent lateral patellar dislocation. J Bone Joint Surg Am. 2016;98(5):417-427.
Orthopedic Research and Reviews

\section{Publish your work in this journal}

Orthopedic Research and Reviews is an international, peer-reviewed, open access journal that focusing on the patho-physiology of the musculoskeletal system, trauma, surgery and other corrective interventions to restore mobility and function. Advances in new technologies, materials, techniques and pharmacological agents are particularly

\section{Dovepress}

welcome. The manuscript management system is completely online and includes a very quick and fair peer-review system, which is all easy to use. Visit http://www.dovepress.com/testimonials.php to read real quotes from published authors. 